\section{LECT URES}

\author{
ON
}

\section{THE PRACTICE OF MEDICINE.}

CHARLES MURCHISON, M.D., LL.D., F.R.S., Physician to St. Thomas's Hospital, and Joint Lecturer on Medicine at St. Thomas's Hospital Medical College; etc.

LeCTURE IV.-Dropsy.-(Concludid.)

ร. Definition.-2. Pathology.-3. Varieties and Diagnosis. General and Local Dropsy. Anasarca. Hydrothorax. Hydropericardium. Ascites. Hydrocephalus. Spurious Dropsies: a. Ovarian; b. Dropsy of Gallbladdir; c. Hydrocele; d. Hydronephrosis ; e. Hydrometra ; f. Acute Edema of Glottis. Classification of true Dropsies: a. Local; b. Par. tial at first, but having a tendency to become general; $c$. General from first. - 4. Prognosis.- 5. Treatment.

Having now described to you the principal varieties of true dropsy, I must call your attention very briefly to certain maladies commonly ranked under the head of dropsy, and designated accordingly, but having in reality a different pathological origin.

a. The most common of these is Ovarian Dropsy. This is not a diropsy in the true sense of the term. It is a cystic growth of the ovary, and has no more right to be called a dropsy than a cystic growth of the kidney, or an hydatid cyst of the liver. All these may fill a great part of the abdomen so as to be mistaken for ascites. This is not the time for considering their points of distinction; but it may be well to state that the fluid in an ovarian cyst is of higher specific gravity (102O-30) than that of dropsy, and that it is often glutinous, or of a brownish or chocolate colour from the admixture of blood.

b. Dropsy of the Gall-Bladder: Hydrops Cystidis Follea.-In cases where the cystic duct becomes obstructed, the bile in the gall-bladder is absorbed, and the gall-bladder itself often undergoes inflammation. When the inflammation is acute, a large quantity of pus may be formed, and the gall-bladder may be converted into one large abscess, which is apt to be mistaken for an abscess of the liver; but when the inflammation is chronic, instead of pus there is sometimes a turbid serous fluid, the quantity of which may amount to several pounds. The resulting tumour may be so large that the swelling has been mistaken for ascites. This is what is known as dropsy of the gall-bladder, but it has always an inflammatory origin, and is not a true dropsy.

c. Hydrocile. - In most systematic works on medicine, hydrocele is referred to as a dropsy of the tunica vaginalis of the testicle, and it is probably the presence of cholesterine in the fluid of hydrocele which has led to this substance being spoken of as a common constituent of dropsical fluid. But hydrocele is in reality not a true dropsy; like dropsy of the gall-bladder, it has always an inflammatory origin. "Its cause," says Chelius, "seems to be a slow inflammation of the vaginal tunic ; frequently it occurs after bruises of the testicle in riding, and the like ; sometimes from cold ; after inflammation or other affections of the urethra ; from wearing ill-fitting trusses, and from syphilis." (System of Surgery. By J. M. Chelius. Translated from the German, by J. F. South, 1847 , vol. ii, p. 498 ).

d. Hydronephrosis, or dropsy of the kidney, is likewise not a true dropsy. It presents itself in two forms. One is a large cystic outgrowth from the cortical substance of the kidney; the other is a saccular dilatation of the kidney itself, consequent on some obstruction or injury of the ureter, and leading, in the first instance, to an enormous accumulation of urine and inflammatory products, and secondarily, to absorption and complete disappearance of the secreting tissue of the organ.

e. Hydrometra, or dropsy of the uterus, is another instance of a spurious dropsy. It is due to a closure of the os uteri, and the consequent distension of the uterus with a catarrhal secretion resembling serum or synovia from its own mucous membrane.

f. Acute Edema of the Glottis is, as its name implies, commonly regarded as a species of dropsy ; but in most cases, at all events, it is an inflammatory affection of the mucous membrane, attended by an unusual amount of serous transudation into the submucous tissue, and imperilling life by closing the entrance to the larynx. It is met with under two conditions : first, as a local result of poisons in the blood, as in erysipelas, typhus, enteric fever, scarlatina, and small-pox; and secondly, as a sequel of some other inflammatory affection in the pharynx or larynx. Its dropsical nature has been argued from the fact that it occurs in certain rare cases of scarlatinal dropsy; but, as a rule, even in scarlatina, it is unassociated with dropsy in other parts of the body.

The morbid conditions to which I have now referred are commonly designated dropsies, but none of them come under the definition of a true dropsy. This is a fact to be remembered, for the distinction is not one merely of name, but of some practical importance. Erroneous opinions respecting the treatment of true dropsies have been founded upon observations made on these spurious dropsies. It is argued, for example, that because certain drugs have little or no effect upon ovarian dropsy, they must be equally inert in all dropsies.

From a clinical point of view, dropsies may be conveniently divided into three classes, viz.:-

A. Those which are local or partial throughout.

B. Those which are local or partial at first, but have a tendency to become general.

c. Those which are general from the first.

A. Dropsies which are Partial or Local Throughout. - The peculiarity of these dropsies is that, however long they last, they are localised in one particular part of the body, and have no tendency to extend beyond. They are all due to venous repletion consequent on some ob. struction to the return of blood to the right side of the heart. The following are some of the principal ways in which these obstructions are brought about.

a. Venous Thrombosis.-By this is meant the formation of a coagulum in the interior of a vein, which adheres to its wall and obstructs its channel. Dropsy of the part which this vein drains of its blood is a common result. This is the pathology of the so-called phlegmasia alba, or white leg, which occurs in the puerperal condition, after severe attacks of fever, and in the advanced stage of phthisis. The œdema of the leg and thigh in these cases is caused by thrombosis of the femoral vein. The much greater frequency of this condition in the left lower extremity than in the right is a fact worth noting, and is probably to be accounted for by the greater length of the left iliac vein, and the circumstance that it has to pass between the right iliac artery and the bone before reaching the vena cava.

$b$. Varicose veins of the thigh or leg are often attended by œdema of the limb below.

$c$. The pressure of enlarged glands in the axilla upon the axillary vein causes œdema of the corresponding arm. In cases of cancer of the mamma, this result is not uncommon.

d. Pressure upon the inferior vena cava or upon both iliac veins by an abdominal tumour, by an enlarged uterus or liver, by accumulations of fæces in the bowels, or even by a great collection of dropsical fluid in the peritoneum, is a common cause of œdema of both lower extrem. ities. In these cases, if the pressure be great and long continued, the epigastric veins in the abdominal wall became greatly enlarged and tortuous, and may be seen through the integuments inosculating above with the mammary veins, which are also enlarged but straighter.

$e$. An intrathoracic tumour-an aneurism, carcinoma, lymphadenoma, or some other form of tumour-may compress the superior vena cava before it enters the right ventricle, and thus give rise to the remarkable phenomenon of dropsy of the upper half of the body, while the lower half remains exempt. In these cases, of which I have chanced to see several, the head, neck, and upper extremities become enormously swollen, livid, and bloated, the integuments are livid, and the eyes prominent and staring. The lower half of the body appears strangely small in proportion to the upper. The jugular veins and the veins of the arms are greatly distended; and, if the pressure be con. tinued for some time, the external mammary veins become greatly enlarged and tortuous, forming a network over the front of the chest, communicating with the veins of the neck and arms and with the epigastric veins, which are also enlarged, but straighter.

$f$. Obstruction of the veins of Galen or of one of the cerebral sinuses may lead to dropsy within the cranium as already stated; but when ossification of the cranium is already complete, hydrocephalus rarely results.

$g$. One of the most common forms of a purely local dropsy is dropsy of the peritoneum or ascites, from obstruction of the circulation through the portal vein or its branches. The causes of obstruction of the portal circulation are very numerous, but may be grouped under four heads, viz. :-

I. Disease of the structure of the liver leading to obliteration of the small branches of the portal vein in its interior, such as occurs in cirrhosis and other forms of chronic atrophy of the organ.

2. Sluggish circulation through the branches of the portal vein in the interior of the liver consequent on some impediment to the circulation of the hepatic vein in diseases of the heart and lungs, or on active congestion of the liver from malaria, or from some local irritation. In 
the former case, however, ascites would only be part of a more general dropsy.

3. Pressure on the trunk of the portal vein or on one of its larger branches by a tumour originating outside the liver, or in the liver itself, by enlarged glands in the fissure of the liver, or by contracting connective tissue, the result of some chronic inflammatory process.

4. Obstruction of the trunk of the portal vein, or of one its large branches, from within, by the formation of a thrombus.

Dropsy from obstruction of the portal circulation is recognised by these characters:-

I. The dropsy commences in the abdomen and is restricted to it. If the lower extremities be implicated, they become so secondarily.

2. If there be dyspnœa, this has followed the ascites and has not preceded it.

3. There are other signs of portal obstruction besides ascites-some more developed in one case, and others in another. These are chiefly : $a$. Enlargement of the superficial veins of the abdomen, especially on the right side and between the sternum and umbilicus ; $b$. A history of hæmorrhoids ; c. Enlargement of the spleen ; $d$. Retching ; e. A tendency to diarrhœa from slight causes ; $f$. Occasionally hæmatemesis or melæna.

4. There are in some cases other indications of disease in the liver, such as pain, tenderness, enlargement, contraction, nodulation of surface, jaundice, etc.

B. Dropsies which are at first Local or Partial, but which have a Tendency to become General.-The dropsy in these cases usually commences in the feet and proceeds upwards, involving at last one or more of the serous cavities. As in the first class, so here, the cause of the dropsy is venous repletion from some obstruction to the flow of blood, but the obstruction is at or near the central organ of the circulation, and hence the dropsies belonging to this class are called cardiac. The dropsy commences in the feet because the veins of the lower extremities, when the patient is erect, having to sustain the weight of a larger superincumbent column of blood, become more turgid than the other veins of the body. In corroboration of this view, you may constantly note that in the early stages of cardiac dropsy the cedema disappears from the feet in the morning, after the patient has maintained the recumbent posture for several hours. But this is not the sole explanation; for you will sometimes see cardiac dropsy commence in the feet even when the patient never leaves his bed. An additional reason is no doubt to be found in the fact, that even in health the circulation in the feet is more sluggish than elsewhere, owing to their greater distance from the heart.

The chief causes of cardiac dropsy are the following :-

I. Disease of the valves on the left side of the heart, and particularly of the mitral valve. Uncomplicated disease of the aortic valves is not often attended by dropsy. Even disease of the mitral orifice may exist for a long time without any dropsy, this only ensuing when the right side of the heart becomes dilated in consequence of the obstruction to the passage of blood through the left side.

2. Chronic diseases of the lungs, and more especially chronic bronchitis and emphysema. These diseases also act by inducing dilatation of the right cavities of the heart.

3. Dilatation of the right side of the heart. This may result, as I have just stated, from- $a$, valvular disease on the left side ; and $b$, disease of the lungs ; but also from $c$, disease of the muscular wall of the right ventricle.

4. Simple weakness in the propelling power of the heart, such as may be observed in fatty or other degenerations of its muscular walls, and in weakened states of the general system consequent on severe attacks of fever, malaria, starvation, hæmorrhages, hyperlactation, chlorosis, etc.

5. Great accumulations of fluid in the pericardium, in cases of pericarditis, by pressing on the inferior vena cava where it enters the right ventricle, occasionally cause dropsy, commencing in the legs,

6. In rare cases, an intrathoracic tumour compresses both the superior and the inferior vena cava, or encroaches upon the right ventricle of the heart, and thus causes dropsy, which may ultimately become general.

c. Dropsies which are General from the first.-In saying that the dropsies of which I now intend to speak are general from the first, I do not mean to imply that from the first there is of necessity dropsy of one or more of the serous cavities, but merely that the œdema of the integuments is not limited on its first appearance to one particular part of the body, and that it appears in the face and trunk as soon as in the legs. From the circumstance that the slightest puffiness of the face materially alters a person's features so as to attract attention, the dropsy in these cases is often first observed in the face, and is thought to commence there. Dropsy answering to this description is almost invariably asso- ciated with a large amount of albumen in the urine and great pallor of the surface, and is due to disease of the secreting tissue of the kidneys. Serious diseases of the kidneys, as we have found, may exist without dropsy, and with but little albumen in the urine; but, when dropsy has a renal origin, the urine usually contains much albumen. I have already explained to you what appears to be the true pathology of renal dropsy. You are to remember that dropsy is chiefly met with in those diseases where the uriniferous tubes become choked up with epithelium, so that the discharge of water from the Malpighian tufts is prevented. In other words, the diseases of the kidneys which mainly give rise to dropsy are acute nephritis and fatty degeneration.

From what I have stated, you will see that general dropsy, whether it be general from the first or not, is the result of either disease of the heart or disease of the kidneys. Every now and then, however, we meet in practice with anomalous cases of general dropsy, in which the urine contains no albumen, and where, on careful examination, no lesion nor functional derangement can be discovered in the heart or lungs. Some of you have recently had an opportunity of seeing a case of this sort in the hospital wards. A man, aged 30 , deaf and dumb from birth, was admitted with general anasarca, which had come on somewhat suddenly, apparently from a chill, a fortnight before. He had been subject to similar attacks all his life, but in the intervals he had been able to follow a laborious calling. The face was bloated and much swollen, but the urine all the time he was in the hospital never contained a trace of albumen, while repeated examinations could discover nothing in the situation, the dimensions, or the sounds of the heart indicative of organic disease ; nor could any sign of disease be detected in the lungs. After a few weeks' treatment by diuretics and iron the dropsy disappeared, the feet only remaining very slightly swollen. It was noted, however, both during and after the dropsy, that the face had a dusky leaden hue, and that the small cutaneous veins of the legs were much dilated. It appeared probable, then, that in this case there was some impediment, probably congenital, to the systemic circulation, in or near the heart, sufficient to maintain a state of repletion of the cutaneous veins, but not sufficient to cause dropsy, except on the superaddition of a chill, by which the state of venous repletion was increased. Other anomalous instances of general dropsy may occur, of which the pathology is even more obscure; but those cases are extremely rare and do not invalidate the general rules which I have laid down for your guidance in diagnosis.

One other remark I have to make in reference to the diagnosis of the cause of dropsy. It is not uncommon for two or more causes to coexist in the same case. We often meet, for example, with disease of the heart and kidneys, with disease of the liver and kidneys, or with disease of all three organs in the same case. But even in these complicated cases the remarks which $I$ have now made will assist you in determining which organ has been mainly instrumental in the production of the dropsy. Thus, when there is evidence that both the kidneys and the heart are diseased, the fact of there being puffiness of the face and pitting of the front of the chest, without much cedema of the legs, would make it probable that the dropsy was mainly renal, an opinion which, of course, would be tested by searching for certain microscopic characters of the urine common in renal dropsies, which we shall have to study together hereafter. Again, when there is ascites in conjunction with oedema of the legs, but when the former greatly preponderates over the latter, or has preceded it, it will be fair to infer that the main cause of the dropsy is in the liver, notwithstanding the presence of valvular disease of the heart.

IV. Prognosis. - The prognosis in dropsy will vary with its cause.

It will be much more favourable in those cases where the cause is re movable, than in those where the primary disease does not admit of cure. The dropsy which is caused by temporary weakness in the propelling power of the heart, resulting from malaria, severe attacks of fever, hæmorrhages, starvation, chlorosis, etc., is the least dangerous and most easily cured. The swelling of the legs dependent upon the pressure of a gravid uterus, or of accumulations of fæces in the bowels, will cease on removal of the pressure ; and the local dropsy of a limb, which is due to thrombosis of the principal vein, often ceases spontaneously on the establishment of a collateral circulation. Acute nephritis is a curable disease, and likewise the so-called "active" or "febrile" dropsy which depends upon it ; and the same remark applies to congestion of the liver and its attendant ascites.

But, even when the cause of the dropsy is irremovable, it does not follow that the dropsy itself cannot be cured. We constantly meet with cases of the most extensive dropsy of the connective tissue and of the serous cavities depending upon incurable disease of the heart or kidneys, where the dropsy is entirely removed and kept away for years. In any such case, however, the prognosis will be bad if the dropsy show no sign of yielding within a week to the recognised methods of treatment. 
The form of dropsy which, as a rule, is least amenable to treatment, and where consequently the prognosis is worst, quoad the dropsy, is ascites depending upon obstructed portal circulation; but such cases, even when the ascites is excessive in amount, are not altogether hopeless. Instances are now and then met with, and have occurred in my own practice, of patients who have recovered from this state, and who have at last permanently lost the dropsy, after repeated operations of paracentesis. More than once also I have known patients seized with excessive ascites, apparently from thrombosis of one of the branches of the portal vein, and after a time completely recover, in all probability from the establishment of a collateral circulation.

The danger of dropsy will depend to some extent on the situation of the liquid. In hydropericardium the danger will be greater than in hydrothorax ; in hydrothorax it will be greater than in ascites; and in œdema of the lungs it will be greater than in œdema of the subcutaneous connective tissue.

Lastly, you will find it stated on good authority that a disappearance of anasarca is often followed by dangerous and even fatal cerebral symptoms. The patient is believed to be getting well, when suddenly he becomes drowsy, stupid, and comatose, and after death the ventricles of the brain are found filled with serous fluid, which is believed to have become suddenly effused there by what is called metastasis from the surface of the body. My experience leads me to doubt the reality of any such danger from the disappearance of dropsy. In the first place, I believe that the occurrence referred to is extremely rare; and secondly, when it does take place, I am inclined to think that the facts admit of a different interpretation. For reasons laid before you in an early part of the lecture, it is highly improbable that dropsical fluid is ever suddenly thrown out in the ventricles of the brain, so as to induce apoplectic symptoms ; it is far more likely that the cerebral symptoms in the cases referred to have been excited by the disease which has given rise to the dropsy, and that the fluid in the ventricles had existed there before the dropsy disappeared from the surface.

v. Treatment. - The rules for the treatment of dropsy are based upon a knowledge of its pathology. The chief objects to be aimed at may be grouped under the following heads.

I. Remove when possible the cause of the dropsy. When dropsy depends upon weakness of the propelling power of the heart, or upon irregularity of its action, these conditions are often remedied by a combination of digitalis and iron, and the dropsy disappears. When it is due to congestion of the liver, or to acute nephritis, the removal of these morbid states will suffice to remove the dropsy. Unfortunately the cause of the dropsy is in many instances irremovable ; we cannot cure valvular disease of the heart, cirrhosis of the liver, or fatty degeneration of the kidneys. Even then, however, the resulting dropsy in many instances admits of removal.

2. Reduce venous repletion. The most direct and certain way of unburdening the loaded veins is blood-letting; but, although this is the immediate effect of blood-letting, it is, in most cases of dropsy, contraindicated, because it tends to weaken the heart and to attenuate the blood, and so favours the transudation of serum outwards through the walls of the vessels wherever the venous current is retarded. Still, in certain cases of cardiac dropsy, where the quantity of blood appears far sreater than the heart can pump through its cavities, a moderate bloodletting often does great good, and materially facilitates the action of other remedies. In cases also of portal obstruction, the application of a few leeches round the anus I have often found to be of signal service : diuretics and purgatives, which before had no effect upon the dropsy, now begin to act. The venous repletion resulting from partial obstruction can also be kept down by purgatives, and especially by those purgatives which increase watery exhalations from the bowel, such as elaterium, jalap, and the sulphates of magnesia and soda. It is in this way that the mineral waters of Carlsbad, Marienbad, Friederichshall, Püllna, Harrogate, and Cheltenham, are so efficacious in cases of portal obstruction. Free diaphoresis and diuresis will also assist in reducing venous repletion; and, in extreme cases of cardiac dropsy, the restriction of the amount of liquid which the patient swallows may contribute to the attainment of the same object.

3. Promote elimination of water through the natural channels of the skin, the kidneys, and the bowels, by means of diaphoretics, diuretics, and purgatives. When elimination by any of these channels is checked, you must endeavour to restore it, while at the same time you excite compensatory exhalations in other directions. You will sometimes hear it stated that diuretics, diaphoretics, and purgatives, have no effect upon dropsies. This is one of the many instances of modern scepticism in the treatment of disease, which, in my opinion, are opposed alike to past observation and present experience. It is very true that you will meet with many cases where these remedies entirely fail ; but it is equally true that you will often succeed in removing large dropsical accumulations by their use.

a. Diaphoretics. - The diaphoretic remedies chiefly resorted to in dropsies are the liquor ammoniæ acetatis, antimony, Dover's powder, the warm bath, and the hot-air bath. Diaphoretics are of most use in renal dropsies, and especially in those which are acute and accompanied by fever. In cardiac dropsies, they are less useful; and in portal dropsy they do no good.

b. Diuretics. - The principal diuretic remedies are the bitartrate, acetate, and nitrate of potash, digitalis, squill, spirit of nitrous ether, scoparium, juniper, copaiba, turpentine, cantharides, and gin. Diuretics are chiefly useful in cardiac and renal dropsies; they have no effect upon portal dropsy. Many instances have come under my notice of extreme cardiac dropsy where a combination of digitalis, squill, and blue pill (one grain of each in pill three times a day), has at once ex. cited a profuse diuresis, followed by a complete and permanent disappearance of the dropsy. Digitalis is a remedy of extraordinary efficacy in many cases of cardiac dropsy, and particularly in cases where the heart's action is irregular, or where it is strong in proportion to the radial pulse. The best form of administering it is a freshly prepared infusion. Scoparium is another useful diuretic in dropsies. It acts most certainly when given in the form of an infusion prepared from freshly gathered "tops"; hence it sometimes succeeds in the hands of the "herbalist", while it fails in those of the regular practitioner. There is a prejudice with many against the use of diuretics in renal dropsy, which I believe to be unfounded. Forty years ago, Bright and Christison recommended a combination of bitartrate of potash and digitalis in this form of dropsy ; and my experience coincides with that of Dr. Gairdner of Glasgow, to the effect that certain diuretics may not only be given with safety in renal dropsy, but that they are, on the whole, more efficacious in this than in any other form of dropsy. When purgatives and diaphoretics fail in renal dropsy, diuretics ought always to be tried; and the best for the purpose are the salts of potash, digitalis, and scoparium. It is a fact worth remembering, that digitalis will sometimes act upon the kidneys when applied to the skin, after both it and other diuretics have failed when given by the mouth. This observation was first made by Sir Robert Christison, and I have repeatedly had occasion to verify it. This mode of employing digitalis is particularly useful when medicines are rejected from irritability of the stomach ; and on several occasions I have known it restore the flow of urine after suppression had set in. Half an ounce of digitalis is to be infused for an hour in a pint of boiling water, and flannel saturated with the infusion is to be wrapped round the abdomen and covered with oiled silk. Minute, but frequent, doses of turpentine or cantharides are believed by many to be efficacious diuretics in renal dropsy. Lastly, copaiba has been recommended as an efficacious diuretic in hepatic dropsy (Transactions of Cinical Society, vol. iii, pp. 26-30); it deserves a trial, but in several instances it has failed in my hands.

c. Purgatives are useful in all forms of dropsy. An intestinal flux often prevents the occurrence of dropsy, and as often removes it after it has become developed. The best purgatives are those which increase the discharge of bile, or promote serous exhalation from the mucous membrane of the bowels. There are calomel and podophyllin, the saline purgatives, jalap, gamboge, and elaterium. Elaterium is the most powerful of these, but it must be given with caution, as its violent action has sometimes a depressing effect upon the heart.

4. Improve the state of the blood and maintain the nutritive processes of the body. Iron is an useful adjunct to the treatment we have been considering in all forms of dropsy; and sometimes you will suc ceed by its means in removing dropsy which has resisted all other methods of treatment. This remark applies even to hepatic dropsy. Dr. Bristowe has recorded several cases of hepatic dropsy treated successfully with quinine and iron, which are well worthy of your attention (Transactions of Clinucal Socicty, vol. ii, p. I2); and I can corro. borate his successful results by several cases which have occurred in my own practice.

5. When, notwithstanding the measures already described, the dropsy continues excessive, have recourse to an operation for its removal. Three operations for this object are practised, viz., paracentesis, acupuncture, and incision. The circumstances under which each of these operations are preferable differ somewhat. Paracentesis or tapping is only had recourse to in ascites, and chiefly in ascites depending upon hepatic disease. The rule is to resort to it when, in spite of internal remedies, the fluid in the peritoneum has risen so high as to interfere with the action of the diaphragm. Too often the operation is merely palliative, and the fluid speedily re-collects ; but now and then a permanent cure of the dropsy, after one or more tappings, is the result. Acupuncture of the legs is frequently resorted to in cardiac dropsy. Three or four punctures in the back part of each leg, with a large needle 
through the skin into the connective tissue beneath, usually suffice. The quantity of fluid which flows from the minute openings is often enormous, and may not merely reduce the bulk of the legs, but also drain the serous cavities. The operation ought not to be too long delayed, for when the integuments became very tense they often slough and give rise to troublesome sores. Acupuncture is also often employed in renal dropsy ; but there is this objection to its use in these cases, that the serum, being loaded with urea or other impurities, is apt to excite inflammation and abscesses at the seats of puncture, which are often followed by erysipelas and sloughing. Under these circumstances, the operation originally recommended many years ago by Dr. Mead, physician to St. Thomas's Hospital, is preferable. This consists in making an incision, about an inch in length in either leg, two finger-breadths above and behind the inner ankle, through the true skin into the subjacent areolar tissue. The wounds may be fomented with cloths moistened with some carbolised lotion.

With these observations I close this lecture; but we shall have to consider the subject of dropsy hereafter when we come to discuss the individual diseases on which it depends.

\section{ENGLISH RE COLLECTION S OF A GERMAN SURGEON.}

Being an Address delivered at St. Thomas's Hospital, May $23 r d, \mathbf{1} 872$.

By DR. L. STROMEYER, Surgeon-General in the North German Army, of Hanover.

Geñlemen, - I suppose I may leave it to the kind care of my youngest English friend, Mr. William Mac Cormac, to account for the liberty I take in addressing you. Let me ask your indulgence for spoiling the Queen's English, which is not my native language. This is the first time that I speak to an English audience. As a surgeon, I dare say, I am not quite a foreigner, having got a sprinkling of English surgery even by inheritance. My father, who was a member of the Royal Medico-Chirurgical Society in London, and well known in his time, by having introduced vaccination in Germany, was a regular pupil of St. Thomas's Hospital, from I 792 to I 793, under Mr. Cline, at a time when Sir Astley Cooper was a demonstrator of anatomy there. He had a very high opinion of English Surgery, and used to say that the best surgeons in the universe were to be found in London; that during a twelve months' presence there he witnessed only a very few cases in which his opinion was different about the propriety of the operations which he daily saw performed. He was able to judge for himself, being already thirty years old when in London, and having been a pupil and assistant of Professor Richter in Göttingen. I followed my father's example, and have been a pupil myself to St. Thomas's Hospital in 1827 and 1828. Mr. Joseph Henry Green introduced me there, and made me acquainted with the splendid circle of surgeons then living in London : Benjamin Travers and John Tyrrell of St. Thomas's Hospital ; Bransby Cooper, Aston Key, and Mr. Morgan, of Guy's Hospital ; William Lawrence and Henry Earle of St. Bartholomew's Hospital ; Sir Benjamin Brodie and Mr. Rose of St. George's Hospital ; Sir Charles Bell of Mid. dlesex Hospital ; Mr. Guthrie of Westminster Hospital ; Mr. Wardrop of Westminster Eye Infirmary. Sir Astley Cooper had already retired to the country. I have only seen him on an occasional visit to St. Thomas's Hospital, where he used to come from time to time when he was tired, as he said, to look after the ewes. It was highly gratifying to see how his presence used to be hailed. The same scene took place when old John Abernethy appeared in St. Bartholomew's Hospital. The students flocked around him, and he generally gave them a speech, in parting, in the open court of the hospital, ending by quoting Shakespeare. Being very partial myself to the great poet, I liked these quotations, which reminded me of Sydenham recommending to read Don Quixote. For a surgeon, nothing is so injurious as dulness; he must always be in good spirits when his services are required. Sir Astley Cooper used to say that a surgeon ought not to read too much; but this, I suppose, meant dull authors, not Shakespeare or Cervantes, who are, both of them, very accurate observers of human nature, like Dickens, Sterne, and Fielding, whose Tom fones, I dare say, you may happen to know.

I could speak for hours if I were to say what influence the surgeons in London whom I have named have had on my mental development. First of all, I admired the truly noble character of the profession, the good feeling of its members to each other, their candour, their humanity in the treatment of severe cases. I can only repeat what my father has said fifty years ago-the operations which I saw were, all of them, necessary, well planned, and, in most cases, executed with great dexterity. Manual dexterity was considered as a quality which scarcely deserved to be mentioned : it was only spoken of where it shone by its absence in a bungling operator. Every operation was executed with the sole view to save the patient's life or to diminish his sufferings, not to show the dexterity of a virtuoso. Circular amputation was preferred to the more showy flap amputation. This I had not forgotten when I had some influence in recommending the circular amputation in times of war, where it is of greater importance still than in chronic cases of civil practice. In 1827, I examined the invalids in Greenwich Hospital, on whom amputation had been performed by the flap method, and found that the fleshy cushion had disappeared entirely. Besides this, I admired English surgery for the simplicity of its application. The great conformity of principles resulting from simplicity struck me as highly valuable, because it makes a deep impression on the mind of a younger member. This conformity gives English surgery a national character. It is not the same in other countries, where only your very particular friends admit that you are right in saying that two and two make four, or that a severe gun-shot fracture of the knee-joint requires amputation.

I was well satisfied with the great caution of English surgeons in adopting innovations. I saw no resections then, and there was no trace of lithotripsy yet. It is better to begin slowly, and then to go on steadily. This is otherwise in Germany and in France, where surgeons are fond of novelties. At present, you may witness the effect of greater caution. Sir Henry Thompson has eclipsed the inventor of lithotripsy, Civiale himself, whose instruments, indeed, were not worth try. ing till Heurteloup had found the right ones for him. Sir William Fergusson, by his articular resections, has surpassed most continental surgeons ; Mr. Spencer Wells, in ovariotomy, all living surgeons.

From what $I$ had observed in London, I came to the conclusion that the beneficial influence of surgery and the high standing of the profession depends chiefly ( $I$ ) on the good feeling of its members towards their patients and towards each other, not excluding those of a former time ; (2) on simplicity ; (3) on a total abnegation of selfishness in plan. ning and executing surgical operations.

You may ask me, gentlemen, why I could not have learned that just as well in Germany. There is no place there which can boast of such a number of great surgeons at the same time. Our greatest capitals have but a few surgeons of eminence in comparison. Whatever may be their merit, their example is not so striking as that of a whole body acting on the same principles. In Paris, the number of surgeons is greater than in our German universities of Berlin or Vienna, but not to be com. pared to London, which I consider as a central point of surgery for the whole globe. This, gentlemen, you may consider as the sincere opinion of a man who has watched the progress of surgery during half a cen. tury. I wish it may remain so for centuries.

After having been in London, I happened to be in Paris at a tíme when Lisfranc was thundering against Dupuytren, whom he used to call "le barbare de la Seine", as a sample of good feeling amongst the profession there. It is one of the great advantages of travelling and of seeing eminent men of other countries that, by observing them in their activity, one may acquire a better notion of their character. Their writings excite greater interest, because we are inclined to give them greater credit. I always admired the simplicity of style in English authors in general, and of surgical writers in particular. Sterne ridicules the pompous style by mentioning the expression of his French barber about the solidity of a new wig : "You may immerse it into the ocean". An Englishman, says Sterne, would have preferred a pail of water. To avoid the barber's style, I took precious good care never to say ocean when I meant a pail of water.

After sketching these general impressions, permit me, gentlemen, to give a few particulars of the manner in which some of my English teachers have influenced me. Having so lately seen one of the greatest battle-fields of modern history, that of Sedan, where I met Mr. William Mac Cormac, who, from over-exertion, did not look quite so well as today, and the siege of Paris afterwards, let me speak of Mr. Guthrie first. I cannot say that I liked him personally quite so well as many of the others; but $I$ admired his energy in maintaining the great principles acquired in the Peninsular war, the necessity of early primary operations, of tying a wounded artery, if possible, on the wounded spot itself. I have done all in my power to keep his doctrines, those of the admirable Hennen, and of old Baron Larrey, in fresh memory since 1848, when the time seemed to approach that Germany must go to war for its own development. For a man of sense, there can be no doubt about the necessity of early primary operations, but in military prac. tice there are difficulties in which it is the duty of every medical man to maintain the sacred cause of humanity. This sentiment was appreciated even by a conqueror like Napoleon I, who said of Larrey that he 